\title{
Micro-Characterization and Three Dimensional Modeling of Very Large Waveguide Arrays by Selective Area Growth for Photonic Integrated Circuits
}

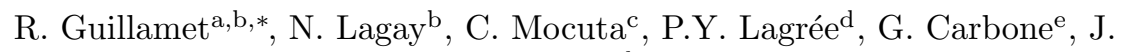 \\ Décobert ${ }^{\mathrm{b}}$ \\ ${ }^{a}$ FOTON-INSA, 20 av. des buttes de Coësmes, 35700 Rennes, France \\ ${ }^{b} I I I-V L a b$, a joint venture between Alcatel-Lucent, Thales and CEA Leti, Route de Nozay, \\ 91460 Marcoussis \\ 'Synchrotron SOLEIL, L'orme des merisiers, 91190 St-Aubin, France \\ ${ }^{d}$ CNRS 8 Univ. Paris 6, UMR 7190, Institut Jean Le Rond d'Alembert, Bô̂te 162, 75005 \\ Paris, France \\ ${ }^{e}$ ESRF, 6 rue Jules Horowitz, 38000 Grenoble, France
}

\begin{abstract}
In this work, selective area growth has been used for the realization of InP based photonic integrated circuits (PIC). To predict the strain, thickness and bandgap energy variations over large and high-density multifunctional arrays, it is necessary to precisely design the shapes and positions of the dielectric masks by computational modeling. To address the mask layout density and complexity in both longitudinal and transversal direction, the use of three dimensional vapor phase model was mandatory. In each SAG region used for individual component processing, the calculated data were compared to experimental ones acquired by synchrotron-based microbeam x-ray diffraction and by micro-photoluminescence wavelength mapping. The excellent result concordance shows that both advanced modeling and characterization techniques are of importance for PIC conception and fabrication.
\end{abstract}

Keywords: A1.Computer simultation, A1.High resolution X-ray diffraction, A2.Selective Epitaxy, B2.Semiconducting III-V materials

*ronan.guillamet@3-5lab.fr 


\section{Introduction}

Selective area growth (SAG) is one of the most attractive techniques to integrate monolithically optoelectronic devices. With this technique, different active and passive structures can be defined simultaneously on dielectric patterned substrates, in a single epitaxial growth step. Since there is no deposition on the inert mask, the growth is restricted to the uncovered areas, leading to a lateral concentration gradient in the vapor phase. Thickness is enhanced in the short vicinity of the masks, generally two stripes surrounding the waveguide direction. In this gap, material composition varies as well, due to sticking coefficient differences between group III elements[1]. In the case of multiple quantum wells (MQW) structures, the SAG effects are advantageously used to tune the band-gap energy wavelength in predetermined areas.

In this work, we investigate a monolithically integrated device made of four separated channels, each one aligning three AlGaInAs-based components: a electro-absorption modulator (EAM), a laser and a monitoring photodiode p-i$\mathrm{n}(\mathrm{PIN})$. Each channel is targeted for emission from $1.470 \mu \mathrm{m}$ (channel 1) up to $1.530 \mu \mathrm{m}$ (channel 4) with $20 \mathrm{~nm}$ wavelength spacing and is laterally separated from the other by a pitch of $200 \mu \mathrm{m}$. The four waveguides are coupled in a semiconductor optical amplifier (SOA), not studied here, via a multi-mode interference (MMI) combiner. This combiner is located in the field area, far from the dielectric and without any mask pad influence and acts as a reference area.

The investigated part of the device totals 13 different areas of interest and for each area, the MQW thickness and composition are different. The corresponding mask layout is presented on Figure 1. In this particularly pattern, each pair of mask stripes is very close but different from its neighbors. This complexity cannot be addressed by a simple bidimensional (2D) model with standard boundary conditions [1]. Several refinements and improvements are required to take into account the periodicity, the size and the influence of the adjacent patterns. As a consequence, advanced micro-characterization techniques have 
been used in precisely positioned areas of the device in order to both refine the model and to adjust the material characteristics.

\section{Experimental Procedure}

Devices structures consisting of AlGaInAs MQW were grown by MOVPE on InP [100] substrate in a commercial AIX200/4 reactor at $650^{\circ} \mathrm{C}$ and 150 mbar. The organo-metallic precursors were TMIn, TMGa and TMAl for element from group III and arsine and phosphine for element from group V . The SAG zones are limited to a small area which requires micro- or submicro- characterization techniques. The emission spectra were measured by micro-photoluminescence ( $\mu$-PL) at room temperature with a $635 \mathrm{~nm}$ laser-diode providing a spot-size of about $1 \mu \mathrm{m}^{2}$. The micro X-ray diffraction[3, 4, 5] ( $\mu$-XRD) was done on the ID01 beamline at European Synchrotron Radiation Facility in Grenoble, France. Using adapted X-ray focusing optics, a sub-micro sized X-ray probe of $0.5 \times 0.5 \mu \mathrm{m}^{2}$ was achieved. This setup has been described in details in reference $[2]$.

The $\mu$-XRD profiles of the samples show satellites peaks on both sides of the substrate Bragg peak $[3,5]$. Figure 2 represents the $\theta / 2 \theta$ scans of the four laser areas. The increase of thickness from channel 1 to channel 4 is clearly observed by the decrease of the peak period. Moreover, the global shift of the peaks toward the small angles indicates a variation of the strain originating from a higher In concentration. By fitting the $\mu$-XRD profiles, we finally determined the thickness and the strain of wells and barriers in the MQW structures. The electronic states of the heterostructures are then calculated with different $\mathrm{Al} / \mathrm{Ga}$ ratio in the well and compared to the measured transition obtained by $\mu$-PL.By coupling these results we calculated the complete AlGaInAs composition in the MQW. The field and the 4 lasers areas composition are reported in table I.

$\mu$-XRD and $\mu$-PL measurements were done on the four EAM, the four lasers, the four PIN and the field area. The measurement of the field parameters is very important as they are used as input parameters of the VPD model. 


\section{Modeling}

SAG simulation commonly uses a vapor phase diffusion (VPD) model, as presented in figure 3 and described in reference [6]. In a two dimensional (2D) classical approach of SAG VPD modeling, the laplacian of the concentration is solved in the $(x, z)$ plane, the calculation domain includes a cut of the two stripes considered as infinite in the longitudinal direction ( $y$ axis) (Fig. 4.a). The boundary side conditions include the periodic reproduction of the same pattern, which is the best way to take into account the long range effect coming from the adjacent neighbors. If we use a $2 \mathrm{D}$ model on the device presented here, two limitations appear: (i) since the stripe width increases, from the EAM up to the photodiode, in the longitudinal direction, the approximation of an infinite stripe should be not valid anymore and reconsidered, (ii) as the stripe neighbors are different, the periodicity is affected in the calculation.

\subsection{Modeling 2D versus $3 D$}

To take into account the variation of mask width along the waveguides, we first compared the 2D model with a full 3D model, which solve the laplacian of the concentration in the $(x, y, z)$ volume. Figure 4 represent the simulated area of these two domain respectively. On figure 4.b, the enlargement of the mask width in the laser zone and the absence of mask in the passive zone induce variations at the center of the waveguide (O point in EAM zone) when compared to the same point on figure 4.a. The concentration gradient is non uniform in the longitudinal direction due to the differences between the Laser, EAM and passive zones. As the different section of the mask are relatively short (between 100 and $600 \mu \mathrm{m})$, these effects should be none negligible.

\subsection{Aperiodic mask}

As presented on figure 1, the width of the mask stripes increase with the channel number. This implies that each channel is surrounded by neighbors different from itself, making inapplicable the standard boundary conditions mentioned above. To take this aperiodicity into account, the calculation domain 
must be extended to the four channels together, which represents the smallest periodic pattern.

\subsection{Boundary conditions}

The standard boundary condition on the sides of the domain is $\frac{d N}{d x}=0$, as presented on figure 3 , which implies that the borders act as mirrors. $N$ is the gaz concentration in the vapor phase and $x$ is a geometrical direction of the dielectric mask. In that case, two consecutive devices would have the following 4321-1234 channel configuration. In the real case of our device, the four channels are reproduced periodically by translation and not by symmetry, leading to a 12341234 configuration. This implies that the boundary conditions on the sides have been changed to $\left.\frac{d N}{d x}\right|_{W_{x} / 2}=\left.\frac{d N}{d x}\right|_{-W_{x} / 2}$ and $N\left(x=-W_{x} / 2\right)=N\left(x=W_{x} / 2\right)$ with $W_{x}$ the size of the domain in the $x$ direction.

\section{Result and discussion}

Figure 5 shows the calculated thickness and PL wavelength of the MQW structure along the waveguide in the middle of the two stripes for channels 1 and 4. For each channel : (i) the three points were calculated using the 2D model, with the corresponding mask width of the EAM, laser and photodiode sections respectively. This supposes an infinite and unique pair of stripes for each calculation. The boundary side conditions imply identical neighbors. (ii) the curve was calculated using the full 3D model on a finite domain in both direction which includes the four channels with their three sections. The boundary conditions are those which report the domain periodically by translation.

One can see that the calculated thicknesses are higher with the $2 \mathrm{D}$ model (points) compared to the 3D model (lines). In the 3D model, as explained above, the absence of mask in the passive zone generates a lower concentration gradient and consequently thinner layers. This effect increases as the mask width increases and consequently the thickness difference is higher for the PIN section than for the EAM one. 
Contrary to what could be expected with thinner quantum wells, the corresponding PL wavelength is not lower but higher. The wavelength increase from one model to another can be explained by the well composition change. Aluminum and gallium, which have the longest diffusion lengths, have an influence that extends further from the mask as compared to indium [6]. Their "longrange effect" $[7]$ has a higher impact with the $2 \mathrm{D}$ model, where the stripes are infinite, and induces aluminum and gallium enrichment. In the 3D model, the stripes are replaced by a passive zone. Aluminum and gallium, which deposits in this zone, are no more available to diffuse in the SAG zone, where consequently the bandgap is lower and the PL wavelength higher.

To take into account these differences and to confirm that the 3D model was as close as possible to the reality, we compared the full calculation of the four channels with the $\mu$-PL and $\mu$-XRD experiments. Figure 6 presents the simulated MQW period thicknesses and PL wavelengths (solid lines) compared with the experimental ones (points) as a function of the position along the waveguide and table I presents the MQW structures of the 4 lasers and the field area measured using $\mu$-PL and $\mu$-XRD compared to the calculated structures using the full 3D model. The very good agreement between simulation and measurements shows the importance of the $3 \mathrm{D}$ calculation, lateral effects and boundary conditions.

\section{Conclusion}

In this work, we have selectively grown a complex and large device, characterized its different sections by $\mu$-PL and $\mu$-XRD and detailed the SAG modelling of these sections. The comparison between two models shows that the 2D model approximation, where the masks are taken as infinite can no longer be considered. A full 3D model, with adapted domain and boundary conditions, is mandatory to precisely describe the mask shape and its influence on the different SAG zones. The very good agreement between measurements and simulations confirmed that this advanced model is fully adapted to the fabrica- 
tion of complex photonic integrated circuits. Furthermore, a complete process has been realized on this device and show very good performances [8].

\section{Acknowledgements}

All the diffractions measurements reported here were preformed on ID01 beamline at ESRF. This study is a part of project ANR-MODULE supported by French government.

\section{References}

[1] M. Gibbon, J.P. Stagg, C.G. Cureton, E.J. Thrush, C.J. Jones, R.E. Mallard, R.E. Pritchards, N. Collis, and A. Chew, Semicond. Sci. Technol., 8:9981010, 1993.

[2] R.Guillamet, N.Lagay, C.Mocuta, G.Carbone, P.Lagrée, J.Décobert, Compound Semiconductor Week (CSW/IPRM), 2011 and 23rd International Conference on Indium Phosphide and Related Materials, 2011

[3] A.A.Sirenko, A.Kazimirov, R.Huang, D.H.Bilderback, S.O'Malley, V.Gupta, K.Bacher, L.J.P.Ketelsen, A. Ougazzaden, Journal of Applied Physics 97 (2005) 063512

[4] L. Mino, A. Agostino, S. Codato, G. Martinez-Criado, C. Lamberti, Nuclear Instruments and Methods in Physics Research Section B: Beam Interactions with Materials and Atoms 284 (2012) 6-9

[5] A.Kazimirov, A.Sirenko, D.H.Bilderback, Z.H.Cai, B.Lai, R.Huang, and A.Ougazzaden, Journal of Physics D: Applied Physics, 39:1422-1426, 2006.

[6] N. Dupuis, J. Decobert, P.-Y. Lagree, N. Lagay, F. Poingt, C. Kazmierski, A. Ramdane, A. Ougazzaden, Journal of Applied Physics 103 (11) (2008) 113113. 
[7] M. A. Alam, R. People, E. Isaacs, C. Y. Kim, K. Evans-Lutterodt, T. Siegrist, T. L. Pernell, J. Vandenberg, S. K. Sputz, S. N. G. Chu, D. V. Lang, L. Smith, and M. S. Hybertsen, Applied Physics Letters, 74(18):26172619, 1999 .

[8] M.Ngo, H.Nguyen, C.Gosset, D.Erasme, Q.Deniel, OFC/NFOEC 2012, 2012. 


\section{Figure 1:}

SAG mask of the device with 4 channels each aligning an EAM, a Laser and a PIN.

The measured points are highlighted by circles.

\section{Figure 2:}

(Color Online) $\theta / 2 \theta$ profiles measured by microbeam X-ray diffraction at the center of each laser area from channel 1 (bottom) to channel 4 (top).

\section{Table I:}

Structures of the field and the 4 lasers areas obtained by simulation and measured by $\mu$-HRXRD and $\mu$-PL.

\section{Figure 3:}

Schematic of the periodic window calculation with governing equations and boundary conditions.

\section{Figure 4:}

Simulation domain for the 2D model (a) and the full 3D model (b). The presented $2 \mathrm{D}$ mask has the same width as that the the EAM area in 3D.

\section{Figure 5:}

(Color online) MQW Thickness (top) and Photoluminescence wavelength (bottom) of the channels 1 and 4 simulated with the 2D (points) and the 3D (lines) models. 


\section{Figure 6:}

(Color online) MQW thickness (top) and Photoluminescence wavelength (bottom) simulated along the four waveguides with the 3D model compared to the measured parameters $(*$ symbols). 


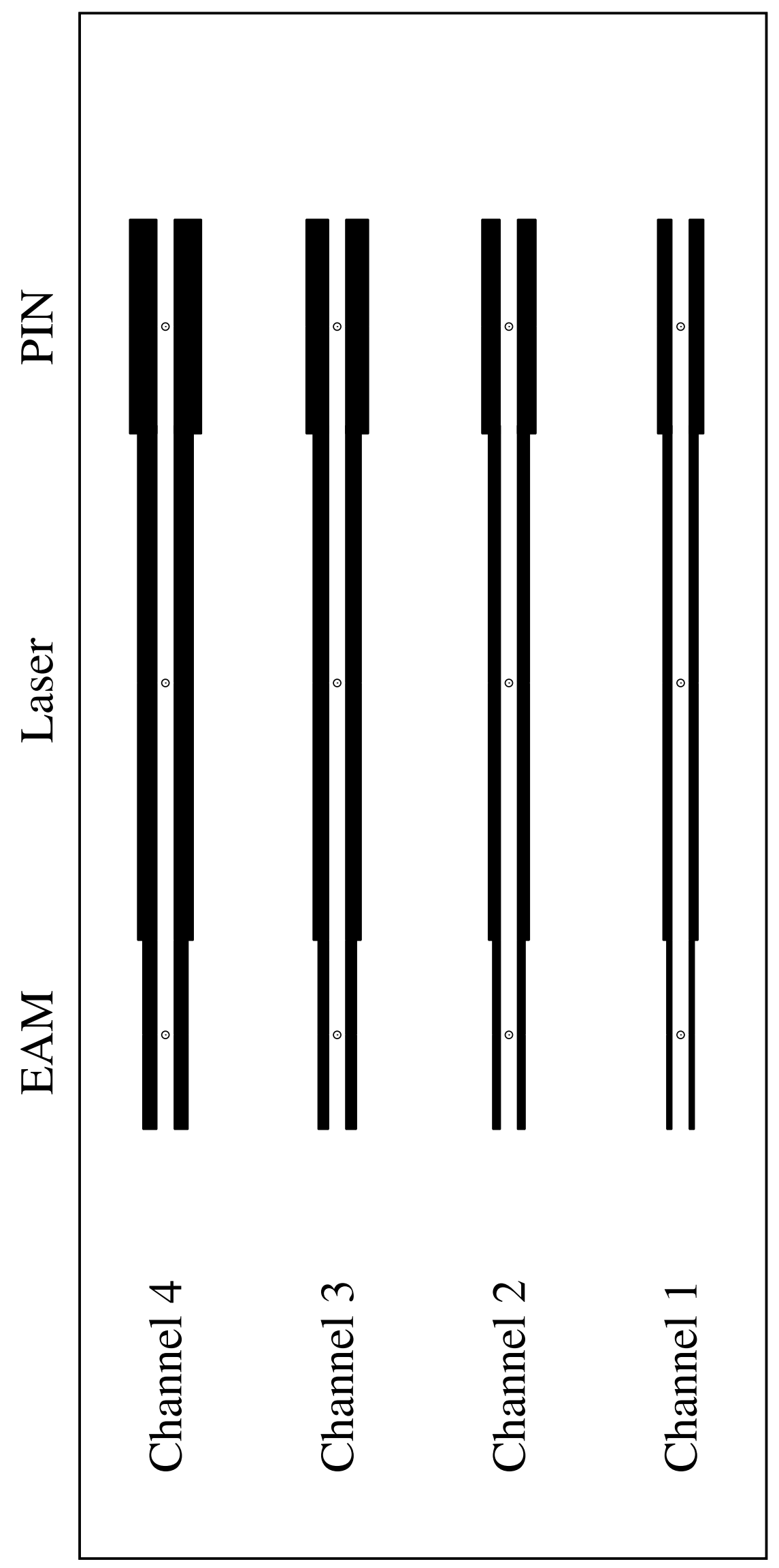




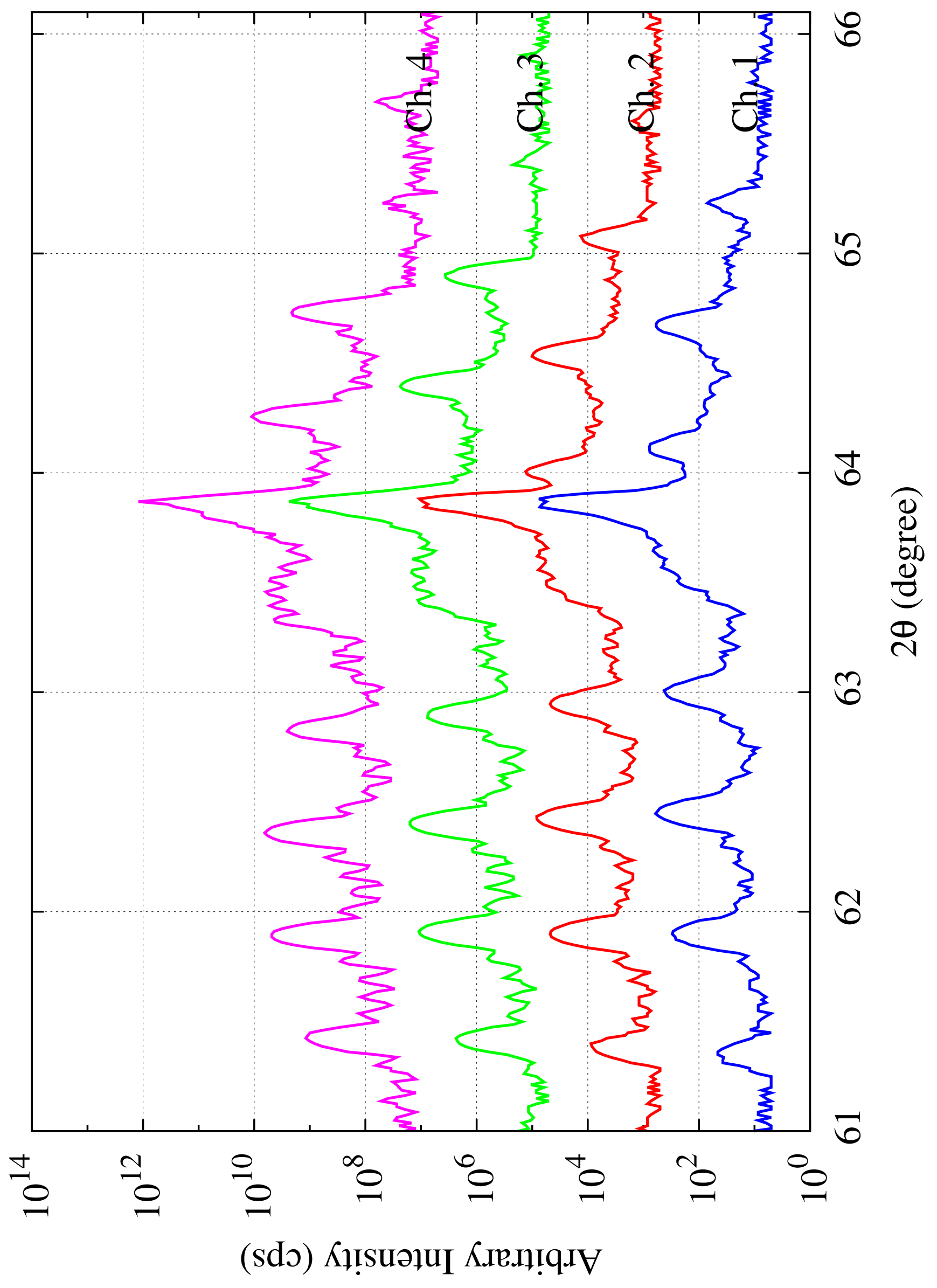




\begin{tabular}{ll|cccc|ccc}
\hline \hline Device & & \multicolumn{3}{|c|}{ Well } & \multicolumn{3}{c}{ Barrier } \\
& & In & Ga & Al & Thic. (nm) & In & Ga+Al & Thic. (nm) \\
\hline \multirow{2}{*}{ Field } & Simulated & 0.670 & 0.217 & 0.113 & 6.11 & 0.44 & 0.56 & 8.40 \\
& Measured & 0.667 & 0.222 & 0.111 & 6.52 & 0.445 & 0.555 & 9.1 \\
\hline \multirow{2}{*}{ Laser 1 } & Simulated & 0.695 & 0.198 & 0.107 & 8.158 & 0.467 & 0.533 & 10.766 \\
& Measured & 0.694 & 0.198 & 0.108 & 7.85 & 0.465 & 0.535 & 10.95 \\
\hline \multirow{2}{*}{ Laser 2 } & Simulated & 0.699 & 0.194 & 0.107 & 8.458 & 0.472 & 0.428 & 11.323 \\
& Measured & 0.7 & 0.193 & 0.107 & 8.5 & 0.47 & 0.53 & 11.2 \\
\hline \multirow{2}{*}{ Laser 3 } & Simulated & 0.704 & 0.190 & 0.106 & 9.024 & 0.476 & 0.524 & 12.033 \\
& Measured & 0.7055 & 0.191 & 0.1035 & 9.1 & 0.475 & 0.525 & 11.8 \\
\hline \multirow{2}{*}{ Laser 4 } & Simulated & 0.709 & 0.186 & 0.105 & 9.583 & 0.482 & 0.518 & 12.730 \\
& Measured & 0.716 & 0.178 & 0.106 & 9.55 & 0.485 & 0.515 & 12.4 \\
\hline \hline
\end{tabular}




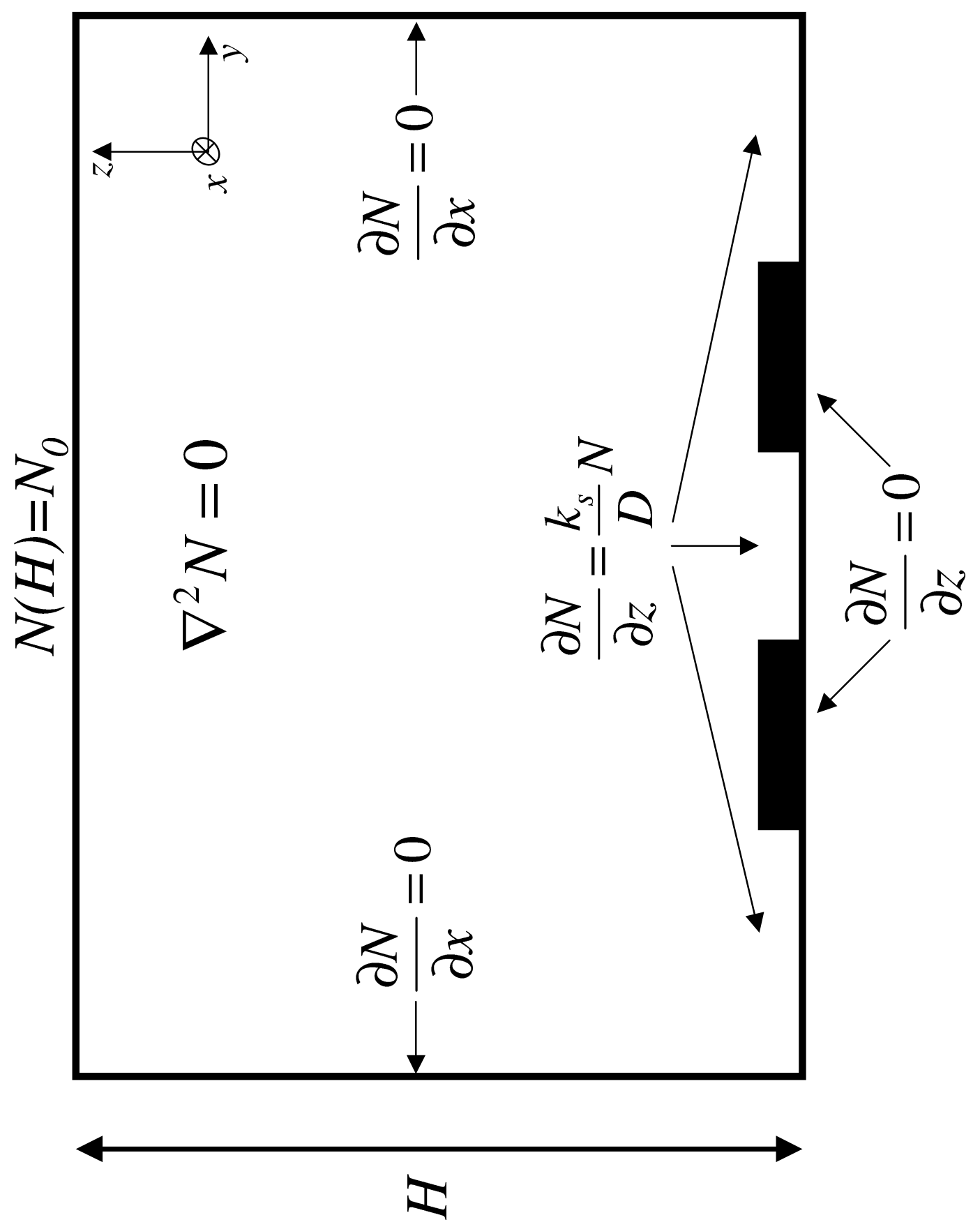




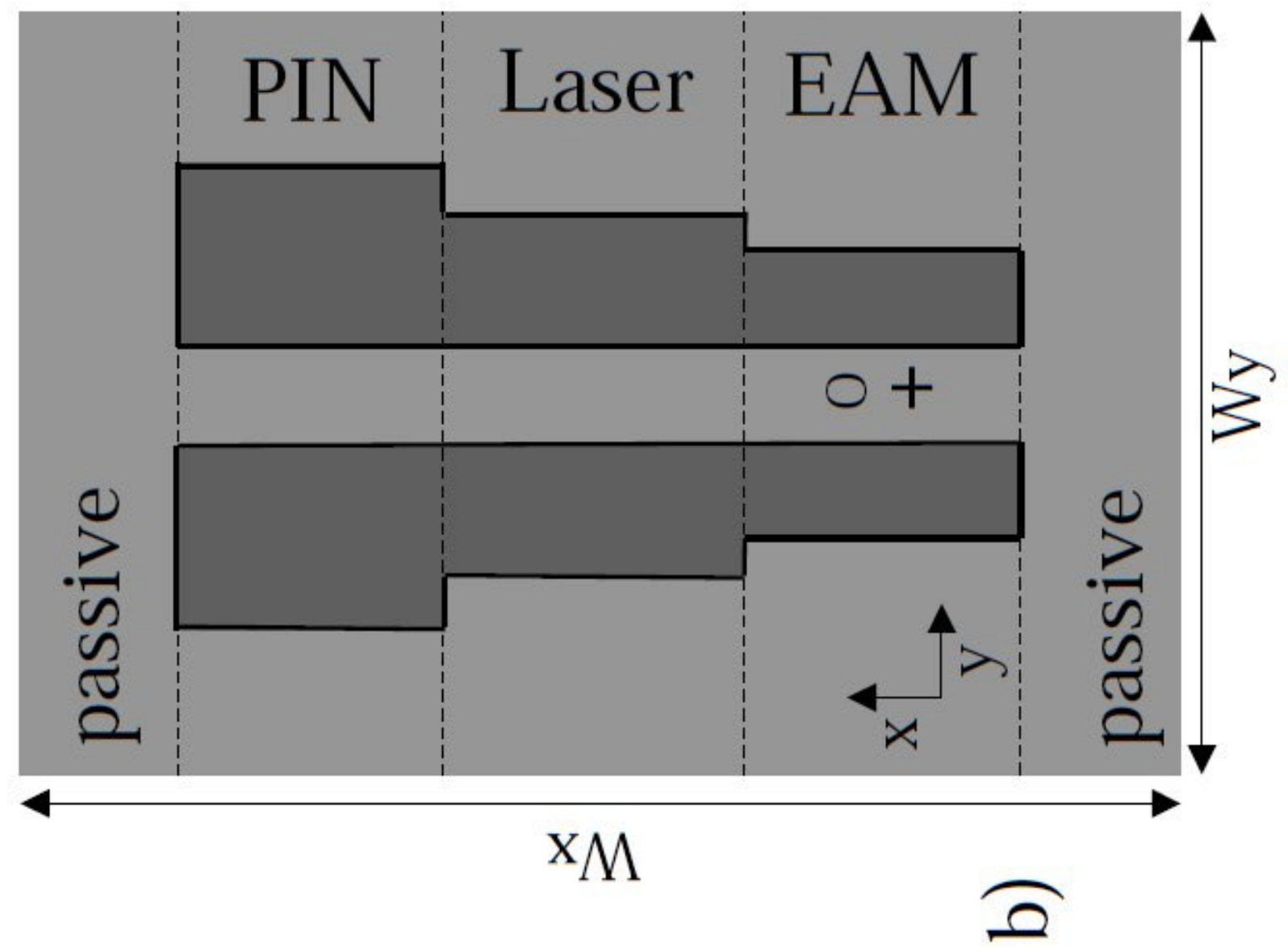

$\measuredangle$

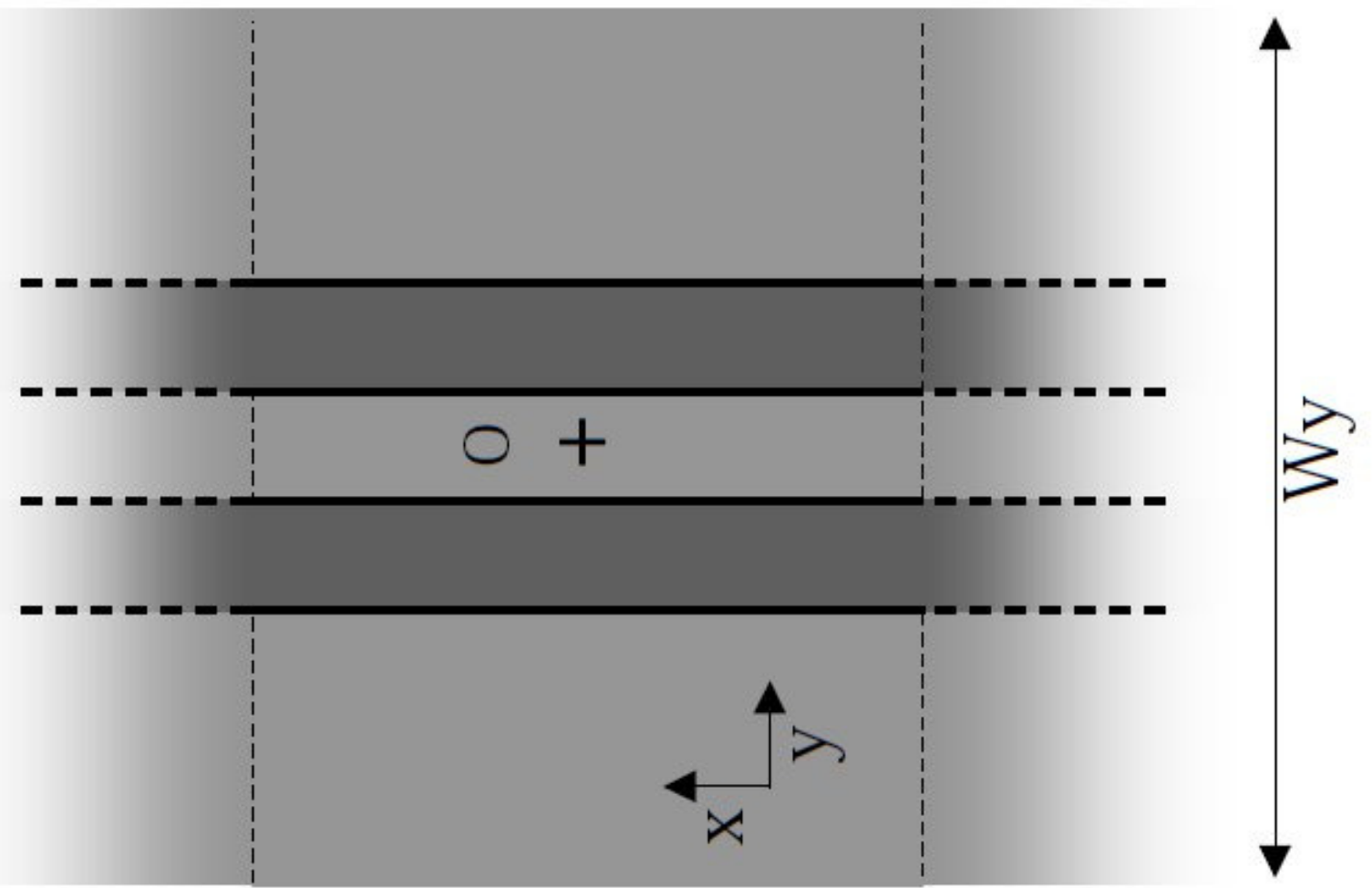



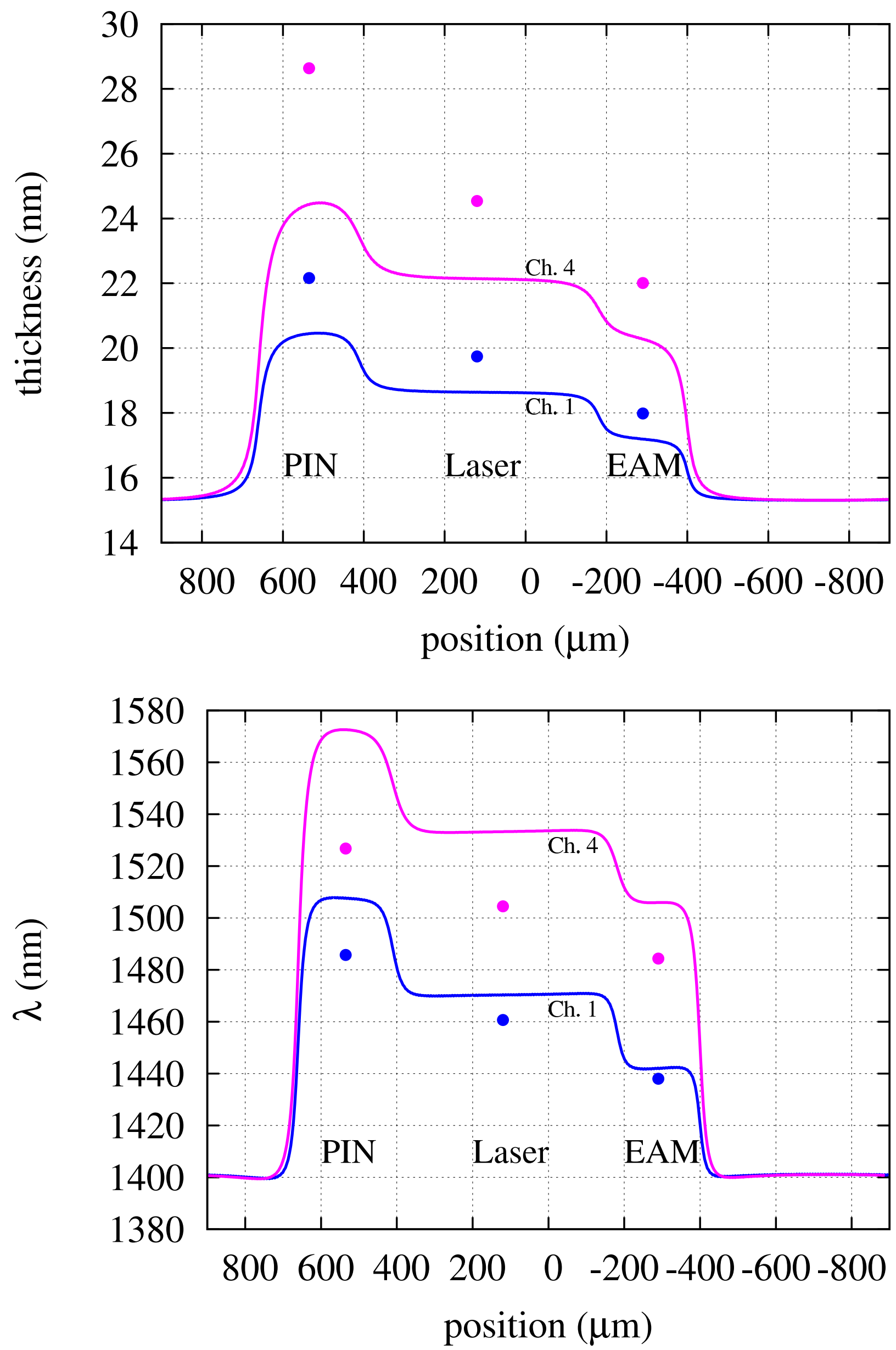

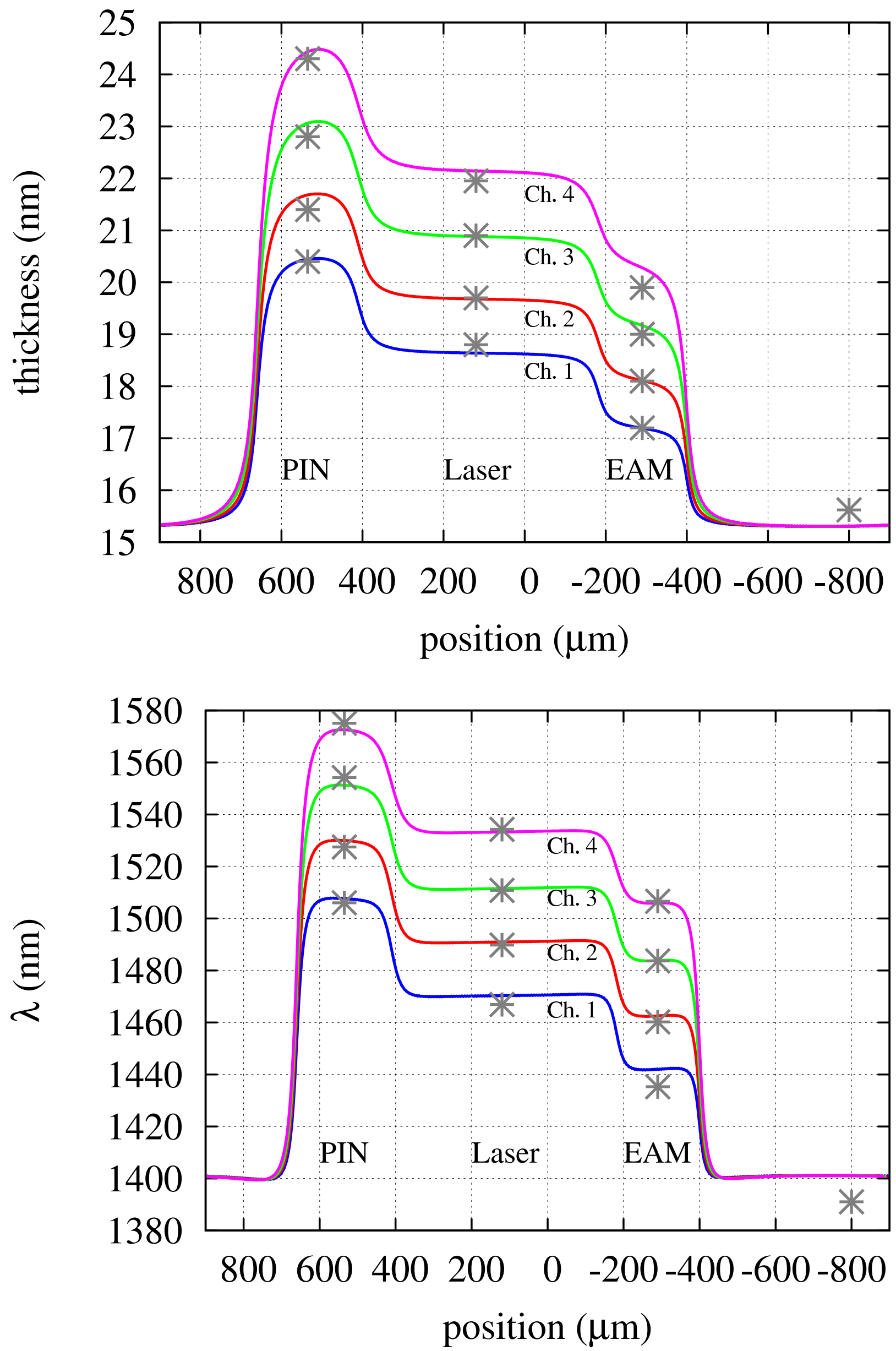